\title{
Bariatrische Chirurgie überzeugt auch noch 5 Jahre postoperativ
}

Fragestellung: Wie sind die Langzeitergebnisse der AdipositasChirurgie bei Diabetikern in kontrolliert-randomisierten Studien?

Hintergrund: Die konventionelle Diabetestherapie ist auf lange Sicht angelegt, scheitert aber oft an der Kooperation der Patienten. Viele übergewichtige Patienten haben bereits häufige Versuche zur Gewichtsabnahme hinter sich, schaffen es einfach nicht und geben resigniert auf. Die Adipositas-Chirurgie bei übergewichtigen Diabetikern ist aber nur bei gesichertem Langzeiterfolg gerechtfertigt.

Patienten und Methoden: Deutlich adipöse Typ-2-Diabetiker (66\% Frauen) erhielten nach Zufallskriterien entweder nur eine intensive konventionelle Therapie oder zusätzlich einen Rouxen-Y-Magenbypass (Verkürzung der Magenpassage) oder eine Magenresektion entlang der kleinen Magenkurvatur (Gastrektomie). Die Patienten waren im Mittel 49 Jahre alt und $44 \%$ insulinpflichtig, die Diabetesdauer betrug 8,4 Jahre, das $\mathrm{HbA}_{1 \mathrm{c}}$

-Wert 9,2 \%, der BMI $37 \mathrm{~kg} / \mathrm{m}^{2}$. Zu Beginn erhielten jeweils 3 von 4 Patienten Lipidsenker (LDL 100 $\mathrm{mg} / \mathrm{dl}$ ) und Antihypertensiva (136/82 $\mathrm{mmHg}$ ).

Ergebnisse: Fünf Jahre postoperativ konnten 134 (89\%) von insgesamt 150 Patienten nachuntersucht werden. Als primärer Endpunkt galt ein $\mathrm{HbA}_{1 \mathrm{c}} \leq 6,0 \%$, das mit der operativen Therapie häufiger erreicht wurde als mit alleiniger medikamentöser Therapie ( $\neg$ Tab. 1). Auch diverse andere Parameter wie Gewicht, Lipidwerte, Blutdruck und Nüchternglukose verringerten sich unter operativer Therapie deutlich und signifikant stärker als unter konventioneller Therapie. Auf antidiabetische Medikamente konnten am Studienende mit konventioneller Therapie nur $2 \%$, mit den operativen Verfahren aber 45 und $25 \%$ der Patienten (mit Bypass signifikant mehr als mit Gastrek-

Um schlank zu werden, ist die bariatrische Chirurgie sicher eine der erfolgreichsten $\mathrm{Me}$ thoden, aber....

Schauer P R, Bhatt D L, Kirwan $J P$ et al. Bariatric Surgery versus Intensive Medical. Therapy for Diabetes - 5-Year Outcomes. N Engl J Med 2017;376:641-51

\begin{tabular}{|c|c|c|c|}
\hline & \multicolumn{3}{|c|}{$\begin{array}{l}\text { Konventionelle intensive antidiabetische } \\
\text { Therapie und... }\end{array}$} \\
\hline & keine Operation & Bypass & Gastrektomie \\
\hline Pat. mit $\mathrm{HbA}_{1 \mathrm{c}} \leq 6,0$ & $5 \%$ & $29 \%$ & $23 \%$ \\
\hline Gewicht (kg) & -5 & -23 & -19 \\
\hline LDL Abnahme (\%) & $-3,7 \%$ & $-12,4 \%$ & $-16,6 \%$ \\
\hline Blutdruck (mmHg) & $-4 /-4$ & $-3 /-6$ & $-8 /-8$ \\
\hline $\begin{array}{l}\text { Patienten ohne } \\
\text { Antidiabetika }\end{array}$ & $2 \%$ & $45 \%$ & $25 \%$ \\
\hline Quelle: Nach Originalie & & & \\
\hline
\end{tabular}

tomie) verzichten. Auch Lipide und Antihypertensiva konnten reduziert bzw. abgesetzt werden.

Schlussfolgerung: Auch noch fünf Jahre nach Adipositas-Chirurgie ist die diabetische Stoffwechsellage im Vergleich zu intensiver medikamentöser Therapie deutlich besser, teilweise sogar normalisiert.

\section{- Kommentar von Prof. Dr. med. H. Holzgreve \\ Operation ersetzt Therapietreue?}

Viele Patienten nehmen nach Abmagerungskuren und nach Fettabsaugung wieder an Gewicht zu. Die vorliegende Analyse einer methodisch zuverlässigen Studie beweist, dass chirurgische Methoden wie Magenbypass und Magenverkleinerung langfristig zu einer erheblichen Gewichtsabnahme um $20 \mathrm{~kg}$ und mehr sowie vielen positiven Veränderungen bei übergewichtigen Diabetikern führen. Die operative, anatomische Veränderung im Bereich des Magens führt zu einer dauerhaften Restriktion der Nahrungsaufnahme, die die Mitarbeit des Patienten bei der Gewichtsreduktion nicht oder nur eingeschränkt erfordert. Allerdings reichen Patientenzahl und Dauer dieser Studie für Analysen zu den typischen diabetischen Komplikationen nicht aus.

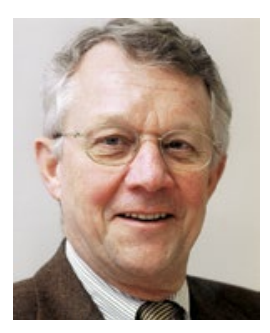

Prof. Dr. med. Heinrich Holzgreve

Internist, Kardiologische Praxis

Burgstr. 7, 80331 München

heinrich.holzgreve@t-online.de 